\title{
Double difference method in deep inelastic neutron scattering on the VESUVIO spectrometer
}

\author{
C. Andreani ${ }^{\mathrm{a}, \mathrm{b}}$, D. Colognesi ${ }^{\mathrm{c}}$, E. Degiorgi ${ }^{\mathrm{b}}$, A. Filabozzi ${ }^{\mathrm{a}, \mathrm{b}}$, M. Nardone ${ }^{\mathrm{d}}$, \\ E. Pace ${ }^{\mathrm{a}, \mathrm{e}}$, A. Pietropaolo ${ }^{\mathrm{a}, \mathrm{b}, *}, \mathrm{R}$. Senesi ${ }^{\mathrm{a}, \mathrm{b}}$ \\ a Università degli Studi di Roma "Tor Vergata", Dipartimento di Fisica, via della Ricerca Scientifica 1, 00133 Rome, Italy \\ ${ }^{\mathrm{b}}$ Istituto Nazionale per la Fisica della Materia, UdR Tor Vergata, via della Ricerca Scientifica 1, 00133 Rome, Italy \\ ${ }^{\mathrm{c}}$ Consiglio Nazionale delle Ricerche, Istituto di Fisica Applicata "Nello Carrara", via Panciatichi 56/30, 50127 Florence, Italy \\ ${ }^{\mathrm{d}}$ Università degli Studi de L'Aquila, Dipartimento di Fisica and Istituto Nazionale per la Fisica della Materia, UdR L'Aquila, \\ via Vettoio 67100, L'Aquila, Italy \\ e Istituto Nazionale di Fisica Nucleare, Sezione di Roma "Tor Vergata", via della Ricerca Scientifica 1, 00133 Rome, Italy
}

Received 18 April 2002; received in revised form 16 September 2002; accepted 3 October 2002

\begin{abstract}
The principles of the Double Difference (DD) method, applied to the neutron spectrometer VESUVIO, are discussed. VESUVIO, an inverse geometry spectrometer operating at the ISIS pulsed neutron source in the $\mathrm{eV}$ energy region, has been specifically designed to measure the single particle dynamical properties in condensed matter. The width of the nuclear resonance of the absorbing filter, used for the neutron energy analysis, provides the most important contribution to the energy resolution of the inverse geometry instruments. In this paper, the DD method, which is based on a linear combination of two measurements recorded with filter foils of the same resonance material but of different thickness, is shown to improve significantly the instrumental energy resolution, as compared with the Single Difference (SD) method. The asymptotic response functions, derived through Monte-Carlo simulations for polycrystalline $\mathrm{Pb}$ and $\mathrm{ZrH}_{2}$ samples, are analysed in both DD and SD methods, and compared with the experimental ones for $\mathrm{Pb}$ sample. The response functions have been modelled for two distinct experimental configurations of the VESUVIO spectrometer, employing ${ }^{6} \mathrm{Li}$-glass neutron detectors and $\mathrm{NaI} \gamma$ detectors revealing the $\gamma$-ray cascade from the $(n, \gamma)$ reaction, respectively. The DD method appears to be an effective experimental procedure for Deep Inelastic Neutron Scattering measurements on VESUVIO spectrometer, since it reduces the experimental resolution of the instrument in both ${ }^{6} \mathrm{Li}$-glass neutron detector and $\gamma$ detector configurations.

(C) 2002 Elsevier Science B.V. All rights reserved.
\end{abstract}

PACS: $28.20 . \mathrm{Fc} ; 28.41 ; 39.30+\mathrm{w} ; 25.60 . \mathrm{Gc}$

Keywords: Neutron absorption; Instrumentation; Spectroscopic technique; Momentum distribution

\footnotetext{
*Corresponding author. Address for correspondence: Dipartimento di Fisica, Universita degli Studi di Roma "Tor Vergata", Via della Ricerca Scientifica 1, Roma 00133, Italy. Tel.: + 39-06-7259-4440; fax: + 39-06-2023-507.

E-mail address: antonino.pietropaolo@roma2.infn.it (A. Pietropaolo).
} 


\section{Introduction}

In the last 20 years the experimental technique for $\mathrm{eV}$ neutron spectroscopy, known as Deep Inelastic Neutron Scattering (DINS) or Neutron Compton Scattering (NCS), has been proved successful in determining single particle physical quantities, such as atomic momentum distributions, $n(p)$, and mean kinetic energies, $\left\langle E_{K}\right\rangle$, for a number of elements, for example, solid and fluid helium $\left({ }^{3} \mathrm{He}\right.$ and $\left.{ }^{4} \mathrm{He}\right)[1]$, other noble gases in both solid and fluid phases [2], molecular systems [3], metals [4], fluid and solid hydrogen [5], glasses [6], graphite [7] and various metal hydrides [8]. These results have been obtained using the eV Spectrometer (eVS), a Resonance Filter Spectrometer (RFS) $[9,10]$, operating at the ISIS pulsed neutron source [11], an instrument which pioneered neutron scattering research at the electron-volt energies in the last decade. This spectrometer was an inverse geometry instrument, [10] specifically designed to make use of the high neutron flux in the eV energy region, produced by the ISIS spallation source at the Rutherford Appleton Laboratory (UK).

The DINS technique exploits the idea, first proposed by Hohenberg and Platzman [12] nearly 30 years ago. According to Hohenberg and Platzman, when the momentum, $\hbar q$, and energy, $\hbar \omega$, transferred from the neutron to a particle (nucleus) are sufficiently large, the dynamic scattering function can be directly related, using the Impulse Approximation (IA) [13], to the single particle momentum distribution of the recoiling particle in its initial state.

In this respect, it is possible to find in DINS conceptual elements and calculation approaches common to other experimental techniques, namely Compton scattering of X-rays and $\gamma$-rays, employed to determine the electron momentum distributions in solids [14], and quasi-elastic electron scattering off nuclei, used to determine nucleon momentum distributions in nuclei [15]. In all the above-mentioned cases, the mathematical formalism for the interpretation of experimental data assumes that the IA is applicable in the description of the scattering process [13]. Deviations from the IA, possibly present at the finite momentum transfer of the neutron scattering experimental set-up, are known as Final State Effects (FSE). These effects appear as an asymmetry in the experimental response function and a shift in the peak position $[13,16]$.

Recently a novel instrument for DINS measurements, the VESUVIO spectrometer, has been installed at the ISIS source [17], upgrading the eVS spectrometer. It has to be stressed that this instrument allows to exploit the ideas originally proposed by Seeger et al. [18] regarding the instrumental energy resolution improvements. As eVS, VESUVIO is an RFS, where the final energy of the scattered neutrons is selected by resonant absorbing filter foils, such as ${ }^{197} \mathrm{Au}$ or ${ }^{238} \mathrm{U}$. It exploits both the intense epithermal neutron flux and the pulsed nature of the source in order to derive $n(\mathbf{p})$ and $\left\langle E_{K}\right\rangle$ quantities. Neutrons with energy matching the resonance energy, $E_{0}$, of the filtering material [18] are strongly captured by the foil over a narrow energy range. The absorbing foils are cycled in and out of the scattered neutron beam and two spectra are recorded: the first with the foil between sample and detectors ( ${ }^{6} \mathrm{Li}$-glass scintillators) and the second with the foil removed [9]. The difference between these two data sets, that is the number of neutrons absorbed by the analysing foil, is the experimental signal which is proportional to the intensity of scattered neutrons at the final energy $E_{0}$. This procedure is known as SD method [19]. The measured total neutron time of flight is used to determine the energy and momentum transfer in the scattering process [9]. With respect to eVS spectrometer, the main improvements of the VESUVIO design are both a better experimental resolution and a wider kinematic space accessible at high energy and momentum transfer in the eV energy region [17]. The first improvement can be achieved by combining filter cooling at liquid $\mathrm{N}_{2}$ temperature and the DD method [18]. In the latter case, the experimental signal is obtained from the weighted difference of two signals recorded in SD method from foils of different thickness (actually, experimental measurements on VESUVIO have been performed so far only using the SD method). The second improvement is achieved owing to a device, allocating ${ }^{6} \mathrm{Li}$-glass detectors and covering a wide 
backscattering angular range [17]. Indeed high values of $\hbar q$ are required to ensure experimental conditions where IA holds with a higher degree of accuracy. This means scattering conditions with high values of $\left|\mathbf{k}_{i}\right|$ and $\left|\mathbf{k}_{\mathrm{f}}\right|, \mathbf{k}_{i}$ and $\mathbf{k}_{\mathrm{f}}$ being the initial and final wave vectors. Unfortunately, the efficiency of the ${ }^{6} \mathrm{Li}$-glass scintillators decreases as the neutron kinetic energy, $E$, increases [20]. To overcome this limitation, an alternative experimental apparatus has been recently proposed for neutron spectroscopy in the eV region on VESUVIO spectrometer: the Resonance Detector Spectrometer (RDS) [20]. The main features and advantages of this apparatus are outlined in Refs. [10,20-24]. In the RDS configuration, VESUVIO is an inverse geometry instrument, where $\gamma$-ray detectors reveal the prompt $\gamma$-ray cascade, produced via $(n, \gamma)$ reaction, after the scattered neutron absorption by the foil. The prompt $\gamma$ detection is used, as previously the case of the neutron detection by ${ }^{6} \mathrm{Li}$-glass scintillators, to assign the total neutron time of flight and the final neutron energy and then to determine energy and momentum transfer. Once the $\gamma$ detector, the foil area and their relative distance have been chosen, the total detection efficiency is independent of neutron energy in the range of interest. Main features and advantages of this experimental configuration have been recently outlined for eVS/ VESUVIO spectrometers, especially in terms of efficiency and background insensitivity of the $\gamma$ detectors in both SD and DD methods [20,24]. It has to be stressed that in the RDS, the DD method requires only one weighted difference between two signals obtained from two foils of different thickness.

Aim of this paper is to present an analysis of the energy resolution function and Monte-Carlo simulations for DINS measurements on VESUVIO spectrometer with SD and DD methods, in both RFS and RDS configurations. In Section 2, basic formulae for DINS measurements on VESUVIO are shown, together with a brief presentation of the instrumental resolution function. In Section 3, the theoretical background for SD and DD methods applied to DINS are described and discussed. In Section 4, Monte-Carlo simulations for VESUVIO in both RFS and RDS configura- tions are presented for reference systems, namely polycrystalline $\mathrm{Pb}$ and $\mathrm{ZrH}_{2}$. Simulations have been performed in both SD and DD methods. In the case of $\mathrm{Pb}$ system the simulated spectra in RDS configuration have been also compared to previous exploratory experimental results in the same configuration, performed on eVS spectrometer. Section 5 will be devoted to discussion and conclusions.

\section{DINS with the VESUVIO spectrometer}

DINS experiments are routinely performed on VESUVIO spectrometer in the IA regime, where the dynamic structure factor is related to the single particle momentum distribution, $n(\mathbf{p})$, by [25]

$S_{\mathrm{IA}}(\mathbf{q}, \omega)=\int n(\mathbf{p}) \delta\left(\omega-\omega_{R}-\frac{\hbar \mathbf{p} \cdot \mathbf{q}}{M}\right) \mathrm{d} \mathbf{p}$

where $n(\mathbf{p})$ is the probability distribution that a particle has momentum $\hbar \mathbf{p}, \hbar \omega_{R}=\hbar^{2} \mathbf{q}^{2} / 2 M$ is the recoil energy of the struck particle of mass $M$ and the $\delta$-function ensures that the kinetic energy is conserved in collisions between neutrons and individual particles. The dynamic structure factor is usually expressed in terms of the West scaling variable, $y$, which represents the component of $\mathbf{p}$ along the direction of $\mathbf{q}$, and is defined by [25]

$y=\frac{M}{\hbar q}\left(\omega-\omega_{R}\right)$.

One can then introduce a longitudinal momentum distribution or Compton profile, $J(y)$, for an isotropic system in terms of $S_{\mathrm{IA}}(\mathbf{q}, \omega)$ :

$J(y)=\frac{\hbar q}{M} S_{\mathrm{IA}}(\mathbf{q}, \omega)$

where $J(y)$ is the probability distribution that a particle has a momentum component of magnitude $\hbar y$ along the direction of $\mathbf{q}$, and is independent of $\mathbf{q}$ at high momentum transfer. It can be shown that, within IA, the Compton profile should be symmetric and should have its maximum at $y=0$ [25]. The single particle mean kinetic energy,

$\left\langle E_{\mathrm{K}}\right\rangle$, is related to the second moment of $J(y)$ via

$\int_{-\infty}^{\infty} y^{2} J(y) \mathrm{d} y=\sigma_{y}^{2}=\frac{2 M}{3 \hbar^{2}}\left\langle E_{\mathrm{K}}\right\rangle$ 
where $\sigma_{y}$ is the standard deviation of the Compton profile.

At high momentum transfers the response function for a fixed-angle detector can be expressed, with a high degree of accuracy, by the following convolution [19]:

$F_{n}(y)=J(y) \otimes R_{n}(y)$

where $n$ is the detector index and $R_{n}(y)$ is the instrument resolution function at the scattering angle 29. Eq. (5) represents the so-called convolution approximation [26], used so far in the data analysis routines for the whole set of experiments on eVS/VESUVIO spectrometers. In a recent paper [27] the validity of the convolution approximation has been reconsidered. However in the present work, it will be shown that data generated via a Monte-Carlo code which does not require the use of the convolution approximation, can be remarkably well described by Eq. (5).

The resolution function, $R_{n}(y)$, consists of two main parts: a geometrical component, arising from the uncertainty in the neutron flight path from moderator to detectors, and a filter component due to the width of the nuclear resonance used for the energy analysis $[19,26,28]$. The former component arises from several distinct contributions (moderator depth, beam size, distance uncertainties, angular acceptance, etc.) [26] and is described by an overall Gaussian functional form with standard deviation, $\sigma_{\mathrm{g}}$. The filter component, at least for thin analyser filters, can be approximately represented by a Voigt function, which derives from the convolution of an intrinsic Lorentzian line shape (i.e., the Breit-Wigner nuclear absorption profile) with a Gaussian Doppler broadening, due to the motion of the atoms in the filter lattice [18]. As a whole, the convolution of the geometrical and filter resolution components still retains a Voigt line shape [28].

As originally suggested by Seeger et al. [18], a significant improvement of the filter resolution can be achieved by using the DD method. In this method, one performs a linear combination of the experimental signals, recorded with two absorbing filters of the same material, but different thickness [18]. This procedure removes almost completely the Lorentzian wings in the filter component of the resolution line shape. It has been suggested that, employing this method on VESUVIO spectrometer at the same level of statistical accuracy of the SD method, a significant reduction in the width in the experimental energy resolution can be achieved [29]. This improvement has been specifically addressed in the construction of the VESUVIO spectrometer and the instrument has been equipped with a rotating device allowing a routinely use of DD method in the backscattering angular range [17].

\section{Theoretical background for SD and DD methods}

\subsection{The filter component of the energy resolution function}

In a DINS experiment, the energy-dependent transmission function of a resonant absorbing filter is given by [30]

$$
T(E)=\exp \left[-\rho \sigma_{\mathrm{eff}} x \bar{f}(E)\right]
$$

where $\bar{f}(E)=f(E) / f\left(E_{0}\right)$ is the normalised nuclear absorption profile, $\sigma_{\text {eff }}$ the effective absorption cross-section at the peak of the resonance, $x$ the thickness and $\rho$ the nuclear density of the filter, respectively.

As previously shown [29] in the case of ${ }^{197} \mathrm{Au}$ and ${ }^{238} \mathrm{U}$ filters, the Breit-Wigner formula can be approximated by a Lorentzian line shape:

$$
L(E)=\frac{1}{\pi} \frac{\Gamma_{0}}{\Gamma_{0}^{2}+\left(E-E_{0}\right)^{2}}
$$

with $E_{0}$ being the resonance energy and $\Gamma_{0}$, independent of $E$, the half-width at half-maximum (HWHM). This expression is assumed to represent the bound-nucleus nuclear absorption profile and it will be considered in the following analytical approach. In the present case, the validity of this expression is ensured by the fact that $\Gamma_{0} / E_{0}$ ratio is lower than 0.02 (see Ref. [18]) for both ${ }^{197} \mathrm{Au}$ and ${ }^{238} \mathrm{U}$ filters.

As far as the Gaussian line shape (accounting for the Doppler broadening) is concerned [18], it can be shown that its standard deviation, $\sigma_{T}$, is proportional to the square root of the analyser effective temperature, $T^{*}$, times the neutron energy 
$E$, i.e.,

$\sigma_{T}=\left(2 \frac{m}{M_{\mathrm{f}}} E T^{*}\right)^{1 / 2}$

where $M_{\mathrm{f}}$ is the nuclear mass of the material composing the filter and $m$ the neutron mass. The quantity $T^{*}$ is approximately related to the Debye temperature of the filter, $\Theta_{\mathrm{D}}$, and to its temperature, $T$, through the formula [18]

$T^{*} \simeq \frac{3}{8} \Theta_{\mathrm{D}} \operatorname{coth}\left(\frac{3}{8} \frac{\Theta_{\mathrm{D}}}{T}\right)$

In a typical VESUVIO scattering experiment, the energy interval of interest is much less than $1 \mathrm{eV}$, around the resonance energy $E_{0}$ (with $E_{0} 4.906 \mathrm{eV}$ for ${ }^{197} \mathrm{Au}$ and $E_{0}=6.671 \mathrm{eV}$ for ${ }^{238} \mathrm{U}$ ). Within this energy interval, the $\sigma_{T}$ dependence on the energy can be neglected. Therefore, as a convolution of the two above line shapes one obtains a Voigt profile:

$$
\begin{aligned}
f(E)= & \frac{1}{\sqrt{2 \pi} \sigma_{T}} \operatorname{Re}\left\{\operatorname{erfc}\left(\frac{\Gamma_{0}-i E+i E_{0}}{\sqrt{2} \sigma_{T}}\right)\right. \\
& \left.\times \exp \left[\left(\Gamma_{0}-i E+i E_{0}\right)^{2} / 2 \sigma_{T}^{2}\right]\right\} \\
f\left(E_{0}\right)= & \frac{1}{\sqrt{2 \pi} \sigma_{T}} \operatorname{erfc}\left(\frac{\Gamma_{0}}{\sqrt{2} \sigma_{T}}\right) \exp \left(\Gamma_{0}^{2} / 2 \sigma_{T}^{2}\right) \\
\equiv & \frac{\psi_{0}}{\pi \Gamma_{0}} .
\end{aligned}
$$

In Eq. (10) the coefficient $\psi_{0}$ defines the ratio of Voigt profile to the corresponding Lorentzian profile peak at $E=E_{0}$. When $\sigma_{T} \rightarrow 0$ (i.e., in the limit $T^{*} \rightarrow 0$ ), then $\psi_{0}=1$, the line shape reduces to a simple Lorentzian:

$f(E)=L(E)$

$f\left(E_{0}\right)=\frac{1}{\pi \Gamma_{0}}$

and $\sigma_{\text {eff }}=\sigma_{0} \psi_{0}$ becomes $\sigma_{0}$, that is the peak absorption cross-section of a bound nucleus.

\subsection{Half-widths}

Defining the dimensionless parameter $\tau=$ $\rho \sigma_{\text {eff }} x$, Eq. (6) can be rewritten as

$T(E)=\exp [-\tau \bar{f}(E)]$ and then the SD transfer function of the spectrometer can be expressed as

$X_{\mathrm{SD}}(E)=1-T(E)$.

In the DD method one makes use of two filters of thickness $x_{1}$ and $x_{2}$, with $x_{2}=x_{1} \beta^{-1}(0 \leqslant \beta \leqslant 1)$ and the instrument transfer function is the result of the following linear combination (see Ref. [18]):

$$
\begin{aligned}
X_{\mathrm{DD}}(E)= & X_{\mathrm{SD}}^{\mathrm{thin}}(E)-\beta X_{\mathrm{SD}}^{\text {thick }}(E) \\
= & \{1-\exp [-\tau \bar{f}(E)]\} \\
& -\beta\{1-\exp [-\tau \bar{f}(E) / \beta]\} .
\end{aligned}
$$

For $\beta=0$ one obtains $X_{\mathrm{DD}}(E)=X_{\mathrm{SD}}(E)$, while in the opposite limit: $\beta=1$ (i.e., two filters of identical thickness), $X_{\mathrm{DD}}(E)$ vanishes identically.

The HWHM for the transfer function $X_{\mathrm{DD}}(E)$, namely $\Gamma_{\mathrm{DD}}(\beta, \tau)$, can be calculated analytically only for $\beta=0$ (SD case) and in the zero effectivetemperature limit $\left(T^{*}=0\right)$, yielding [18]

$$
\begin{aligned}
& \lim _{T^{*} \rightarrow 0} \Gamma_{\mathrm{DD}}(0, \tau) \\
& \quad=\Gamma_{\mathrm{SD}}^{0}(\tau)=\Gamma_{0}\left\{\frac{\tau}{\ln [2 /(1+\exp (-\tau))]}-1\right\}^{1 / 2}
\end{aligned}
$$

where the superscript 0 marks the absence of any Doppler broadening. The growth of $\Gamma_{\mathrm{SD}}^{0}(\tau)$ with increasing $\tau$ is shown by the full line in Fig. 1.

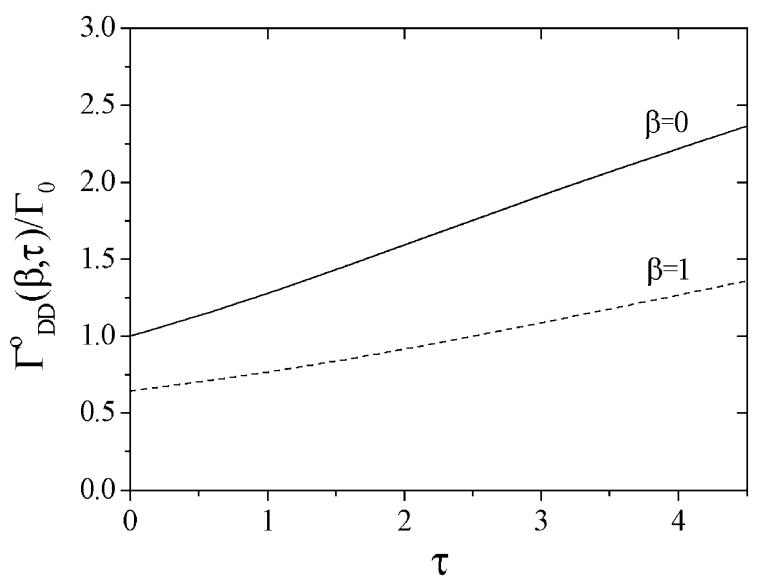

Fig. 1. Ratio between the HWHM of the DD transfer function in the zero temperature limit, $\Gamma_{\mathrm{DD}}^{0}(\beta, \tau)$, and the Breit-Wigner HWHM, $\Gamma_{0}$, for both $\beta=0$ (full line) and $\beta=1$ (dashed line). Notice that, in the $\beta=0$ case, $\Gamma_{\mathrm{DD}}^{0}(\beta, \tau)$ coincides with the SD half-width in the absence of Doppler broadening, given by Eq. (15). 
Values of $\Gamma_{\mathrm{SD}}(\tau)$ for non-zero effective temperatures have to be evaluated numerically, starting from Eq. (13).

When $\beta \neq 0$, the HWHM of the transfer function $X_{\mathrm{DD}}(E)$ must be calculated numerically, even in the $T^{*} \rightarrow 0$ limit. A significant behavior of $\Gamma_{\mathrm{DD}}(\beta, \tau)$ can be learned in the limiting case of very large $\beta$ (namely $\beta \rightarrow 1$ ). Indeed, expanding Eq. (14) around $\beta=1$, one obtains

$$
\begin{aligned}
X_{\mathrm{DD}}(E) \simeq & (1-\beta)\{1-\exp [-\tau \bar{f}(E)] \\
& -\tau \bar{f}(E) \exp [-\tau \bar{f}(E)]\} .
\end{aligned}
$$

This transfer function, which vanishes for $\beta=1$, has therefore a limiting line shape which is independent of $\beta$. Its HWHM is, in the zero effective-temperature limit, a smoothly varying function of $\tau, \Gamma_{\mathrm{DD}}^{0}(\beta \rightarrow 1, \tau)$, which can be evaluated numerically, using the above Eq. (16). The ratio between $\Gamma_{\mathrm{DD}}^{0}(\beta, \tau)$ and $\Gamma_{0}$ is reported as a function of $\tau$ in Fig. 1, for both $\beta=0$ and 1 . Intermediate $\beta$ values will yield values falling between these two curves for any given value $\tau$ of the thinner filter.

It is worth noting that the value of $\Gamma_{\mathrm{DD}}^{0}(1, \tau)$ when $\tau \simeq 0$ represents, for a given value of $\Gamma_{0}$, the minimum attainable HWHM. This situation corresponds to a DD performed using two identical, sufficiently thin filters. The corresponding transfer function can be worked out analytically expanding the exponential in Eq. (16) to obtain

$$
\lim _{\tau \rightarrow 0 ; \beta \rightarrow 1} X_{\mathrm{DD}}(E)=\frac{1}{2}(1-\beta) \tau^{2} \bar{f}(E)^{2} .
$$

In the zero effective-temperature limit the squared Lorentzian in Eq. (17) has an HWHM given by

$\Gamma_{\mathrm{DD}}^{0}(1,0)=\Gamma_{0} \sqrt{\sqrt{2}-1}=0.64 \Gamma_{0}$.

In Fig. 2, we report numerical calculations of $\Gamma_{\mathrm{DD}}(\beta, \tau) / \Gamma_{0}$ ratio for ${ }^{197} \mathrm{Au}$ and ${ }^{238} \mathrm{U}$ filters at room temperature, obtained in both $\beta=0$ and 1 limits. In this figure, the $\beta=0$ limit corresponds to a SD spectrum with a filter of thickness $\tau$, while the $\beta=1$ limit corresponds to a DD spectrum obtained using two identical filters of thickness $\tau$. All intermediate $\beta$ values yield curves laying between these two cases. An example of the dependence of $\Gamma_{\mathrm{DD}}(\beta, \tau)$ on $\beta$ is shown in Fig. 3

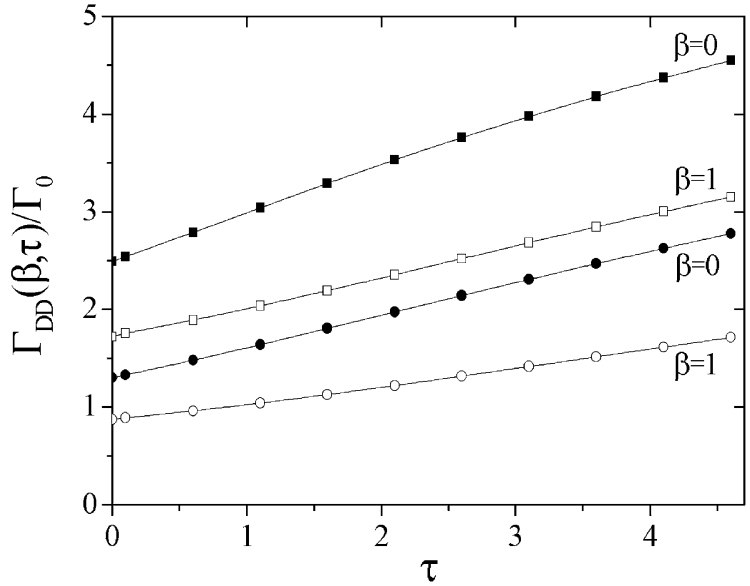

Fig. 2. Ratio between the HWHM $\Gamma_{\mathrm{DD}}(\beta, \tau)$ and $\Gamma_{0}$, calculated for a ${ }^{197} \mathrm{Au}$ filter foil at room temperature (circles) and for ${ }^{238} \mathrm{U}$ at $T=77 \mathrm{~K}$ (squares), both for $\beta=0$ (full symbols) and $\beta=1$ (empty symbols). Lines are only eye-guides.

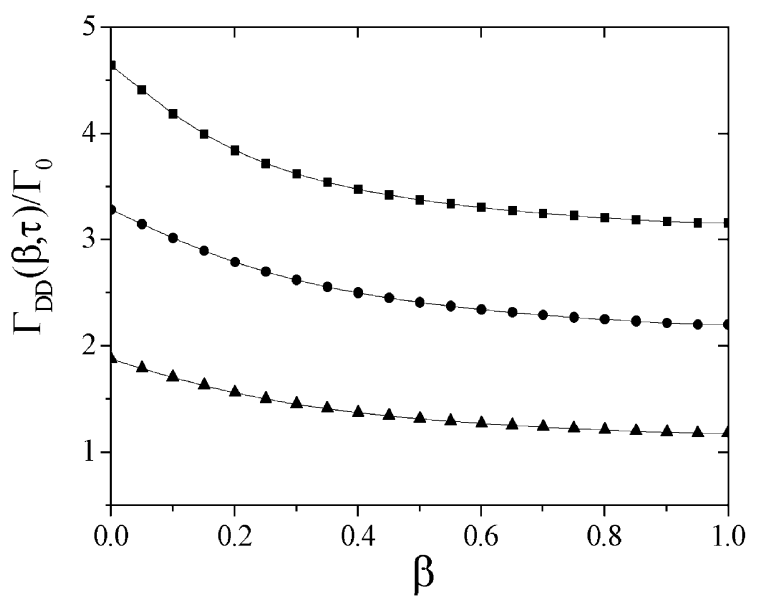

Fig. 3. Ratio between the HWHM $\Gamma_{\mathrm{DD}}(\beta, \tau)$ and $\Gamma_{0}$ as a function of $\beta$ for the ${ }^{238} \mathrm{U}$ foil (squares at room temperature and circles at liquid nitrogen temperature) and the ${ }^{197} \mathrm{Au}$ foil at room temperature (triangles) for the $\tau$ values reported in Table 1. Lines are only a guide for eyes.

for the aforementioned filters at the $\tau$ values reported in Table 1.

The main advantages of DD method are a sensitive reduction of the resolution and a significant qualitative change in the shape of the Lorentzian wings of the transmission function. This occurs since the wings of $X_{\mathrm{SD}}(E)$ are 
independent of $\tau$, except for multiplication by $\tau$ (whenever $\bar{f}(E)$ is small, the expansion of the exponential yields $\bar{f}(E)$ itself). As a consequence, the terms of the DD linear combination in Eq. (14)

Table 1

Most significant parameters for typical gold and uranium foils in SD and DD configurations

\begin{tabular}{llll}
\hline & $\mathrm{Au} @ 300 \mathrm{~K}$ & $\mathrm{U} @ 300 \mathrm{~K}$ & $\mathrm{U} @ 77 \mathrm{~K}$ \\
\hline$E_{0}(\mathrm{eV})$ & 4.906 & 6.671 & 6.671 \\
$\Gamma_{0}(\mathrm{meV})$ & 70 & 14 & 14 \\
Thickness $(\mu \mathrm{m})$ & 10 & 30 & 30 \\
$\psi_{0}$ & 0.8356 & 0.3460 & 0.5036 \\
$\sigma_{\text {eff }}($ barn $)$ & 30665 & 7543 & 10980 \\
$\tau$ & 1.8 & 1.1 & 1.6 \\
$\Gamma_{\mathrm{SD}}(\tau)(\mathrm{meV})$ & 130 & 64 & 45 \\
$\Gamma_{\mathrm{SD}}(\tau=0)(\mathrm{meV})$ & 90 & 53 & 34 \\
$I_{\text {peak }}^{\mathrm{SD}}$ & 0.84 & 0.66 & 0.79 \\
$\Delta_{I}^{\mathrm{SD}}$ & 1.2 & 1.6 & 1.3 \\
$\beta$ & 0.28 & 0.28 & 0.28 \\
$\Gamma_{\mathrm{DD}}(\beta, \tau)(\mathrm{meV})$ & 102 & 50 & 36 \\
$I_{\text {peak }}^{\mathrm{DD}}$ & 0.56 & 0.39 & 0.52 \\
$\Delta_{I}^{\mathrm{DD}}$ & 1.3 & 2.0 & 1.5 \\
\hline
\end{tabular}

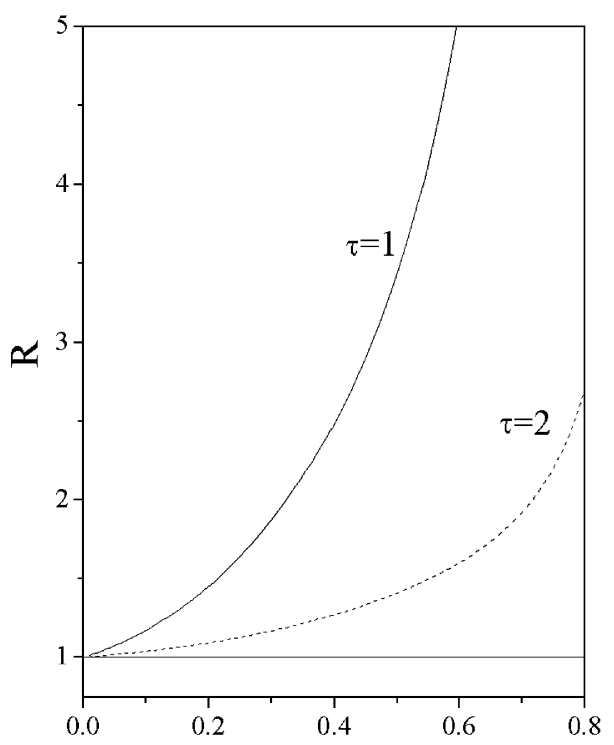

(a)

$\beta$ partially cancel out with a consistent reduction of the $X_{\mathrm{DD}}(E)$ wings.

\subsection{Counting statistics}

In the scattering experiments performed on VESUVIO an incident flux of neutrons characterised by a white energy spectrum is scattered from a sample. The scattering peak intensity, $I_{\text {peak }}^{\mathrm{SD}}$, and its corresponding statistical uncertainty, $\sigma_{I}$, can be easily calculated in terms of same quantities, $I_{\text {out }}$ and $\sigma_{\text {out }}$, measured in the absence of the filter. We also introduce the relative statistical uncertainties $\Delta_{I}^{\mathrm{SD}}=\sqrt{\sigma_{I}^{2} /\left(I_{\text {peak }}^{\mathrm{SD}}\right)^{2}}$ and $\Delta_{\text {out }}=$ $\sqrt{\sigma_{\text {out }}^{2} / I_{\text {out }}^{2}}$ for the SD and the filter-out cases, respectively. Assuming that two independent measurements are performed using the same counting time, one finds

$I_{\text {peak }}^{\mathrm{SD}}=I_{\text {out }}-I_{\text {in }}=I_{\text {out }}\left(1-\mathrm{e}^{-\tau}\right)$

with an estimated statistical uncertainty given by

$\sigma_{I}^{2}=\sigma_{\text {out }}^{2}+\sigma_{\text {in }}^{2}=\sigma_{\text {out }}^{2}\left(1+\mathrm{e}^{-2 \tau}\right)$

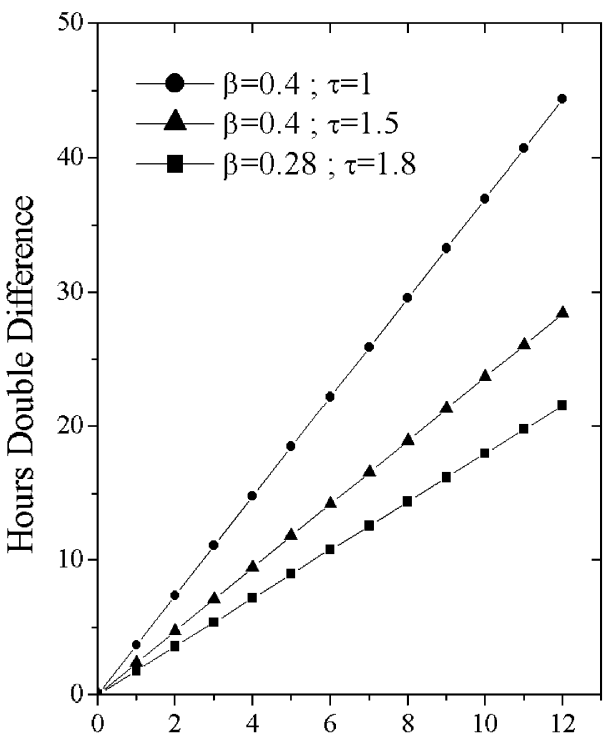

(b)

Hours Single Difference

Fig. 4. (a) Time factor $R$ as a function of $\beta$ for $\tau=1$ (full line) and $\tau=2$ (dashed line); (b) relationships between the time required in $\mathrm{DD}$ and SD experiments for the same statistical accuracy for three couples of $\tau$ and $\beta$ values. 
and, therefore, a relative statistical uncertainty expressed by

$\Delta_{I}^{\mathrm{SD}}=\sqrt{\frac{\sigma_{I}^{2}}{\left(I_{\text {peak }}^{\mathrm{SD}}\right)^{2}}}=\Delta_{\text {out }} \sqrt{\frac{1+\mathrm{e}^{-2 \tau}}{\left(1-\mathrm{e}^{-\tau}\right)^{2}}}$.

Let us now deal with the DD method, where three independent measurements are needed. On VESUVIO these measurements are performed with the same counting time. The peak intensity, $I_{\text {peak }}^{\mathrm{DD}}$, is then expressed by

$I_{\text {peak }}^{\mathrm{DD}}=I_{\text {out }}\left(1-\beta-\mathrm{e}^{-\tau}+\beta \mathrm{e}^{-\tau / \beta}\right)$

with an associate relative statistical uncertainty, $\Delta_{I}^{\mathrm{DD}}$ :

$\Delta_{I}^{\mathrm{DD}}=\Delta_{\text {out }} \sqrt{\frac{(1-\beta)^{2}+\left(\mathrm{e}^{-\tau}\right)^{2}+\left(\beta \mathrm{e}^{-\tau / \beta}\right)^{2}}{\left(1-\beta-\mathrm{e}^{-\tau}+\beta \mathrm{e}^{-\tau / \beta}\right)^{2}}}$.

The ratio $R=\left(\Delta_{I}^{\mathrm{DD}} / \Delta_{I}^{\mathrm{SD}}\right)^{2}$ is the time factor required for each single measurement (under the reasonable assumption of a Poisson counting statistics) to obtain the same statistical accuracy achieved in an SD spectrum. The total time factor turns out to be $\frac{3}{2} R$, since DD needs three measurements and SD only two. Fig. 4(a) shows the ratio $R$ as a function of $\beta$, while in Fig. 4(b) the time required in a DD experiment to obtain the same statistical accuracy as in an SD one is reported for three couples of $\tau$ and $\beta$ values. It is clear that larger values of $\beta$ produce narrower transmission functions at the expense of a decreased counting rate. In Table 1 the most important features (width, peak intensity and statistical uncertainty) of typical filters $(10 \mu \mathrm{m}$ thick ${ }^{197} \mathrm{Au}$ and $30 \mu \mathrm{m}$ thick ${ }^{238} \mathrm{U}$ ) available on VESUVIO spectrometer are reported. Furthermore the corresponding features for the DD method are reported for the value $\beta=0.28$, which represents an optimized configuration, providing the best compromise between counting time and resolution improvement, as shown in Ref. [18].

\section{Monte-Carlo simulations and experiments with SD and DD methods}

In order to test the effectiveness of a complete VESUVIO experiment, from the instrument con- figuration to the data acquisition and analysis, simulations of standard reference experiments were carried out using a Monte-Carlo approach. The Monte-Carlo code employed for simulations is a modification of the DINSMS code described in Ref. [31], which was specifically developed to simulate the performance of the eVS spectrometer for NCS from condensed matter, and has been successfully applied to evaluate single and multiple scattering contributions from solid samples in conventional SD experiments. DINSMS code takes into account the specific features of neutron source, filters and detectors present on eVS/VESUVIO and assumes the scattering process to be described within the framework of the IA.

\subsection{Simulated DINS experiments with ${ }^{6}$ Li-glass detectors}

Simulations of typical DINS measurements on VESUVIO spectrometer using ${ }^{6} \mathrm{Li}$-glass detectors on two different reference samples $\left(\mathrm{Pb}\right.$ and $\left.\mathrm{ZrH}_{2}\right)$, both employing SD and DD methods, are presented in this section. In order to accomplish the simulations for the DD method, the DINSMS code has been modified to incorporate two distinct, SD transfer functions, $X_{\mathrm{SD}}(E)$, for thin $\left(\tau_{1}=1.8\right)$ and thick $\left(\tau_{2}=6.4\right)$ natural uranium foils at room temperature. These values correspond to the aforementioned optimized foil thickness ratio $\beta=0.28$. The simulation has been performed using a detector configuration such as to simulate forward scattering experiments at a single fixed angle of $2 \vartheta_{n}=42.54^{\circ}$.

Taking advantage of the dominant contribution of the resolution to the $\mathrm{Pb}$ response function, $\mathrm{Pb}$ calibration samples are routinely used on eVS/ VESUVIO spectrometer with the aim to determine the geometric and filter components of the resolution function. To this aim a Monte-Carlo simulation with SD method for a polycrystalline $\mathrm{Pb}$ sample at room temperature has been performed. The corresponding scaling function, obtained as explained in Ref. [31], is reported in Fig. 5 for the ${ }^{238} \mathrm{U}$ foil with $\tau=1.8$. The $\mathrm{Pb}$ momentum distribution is very well approximated by a Gaussian function and the reference value of 


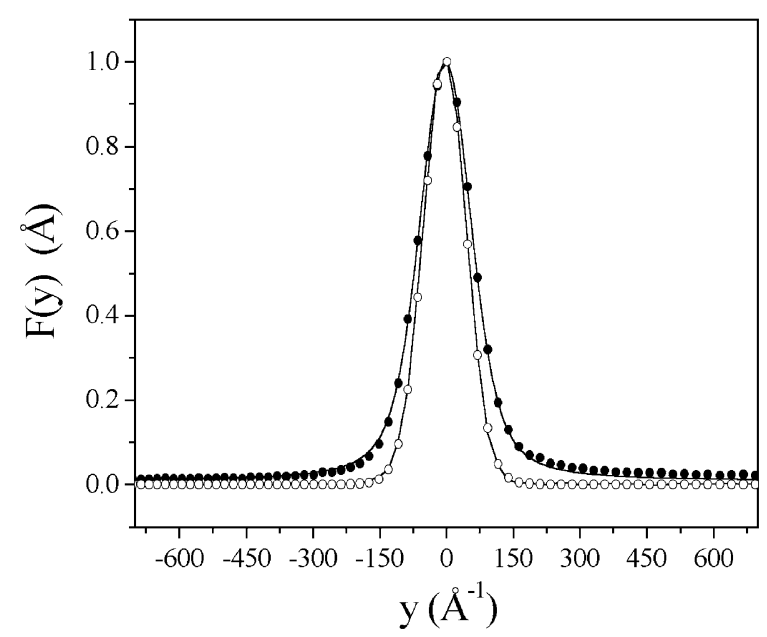

Fig. 5. Normalised SD (full circles) and DD (open circles) simulations of the response function $F(y)$ for polycrystalline $\mathrm{Pb}$ sample, obtained employing ${ }^{238} \mathrm{U}$ as analyser foil with $\tau=1.8$ and $\beta=0.28$ at $T=300 \mathrm{~K}$. The simulations refer to a scattering angle $2 \vartheta_{n} \simeq 42.54^{\circ}$. Full lines are the corresponding fits, according to Eqs. (24) and (25).

for the standard deviation, $\sigma_{\mathrm{Pb}}=35.3 \AA^{-1}$, of this distribution has been used in the simulation [19]. The simulation with DD method is also reported in Fig. 5, where the expected resolution improvement is clear.

The final simulation involves scattering from hydrogenous sample. In this case, a room temperature polycrystalline $\mathrm{ZrH}_{2}$ sample has been chosen, which is well known theoretically and has been the object of several measurements on eVS [32]. The interatomic potential acting on protons in $\mathrm{ZrH}_{2}$ is very well approximated by a harmonic one and therefore the corresponding proton momentum distribution has been assumed to be a Gaussian function. A value for the standard deviation, $\sigma_{\mathrm{H}}$, equal to $4.14 \AA^{-1}$ has been selected [32]. Two simulations have been performed, both using the SD instrument transfer function, but with thin and thick foils, respectively. The DD spectrum was finally obtained carrying out the linear combination of the two SD simulations, with coefficient $\beta$ (see Eq. (14)). The response function, obtained transforming the simulated time-of-flight spectra according to the West scaling, is shown in Fig. 6 for ${ }^{238} \mathrm{U}$.

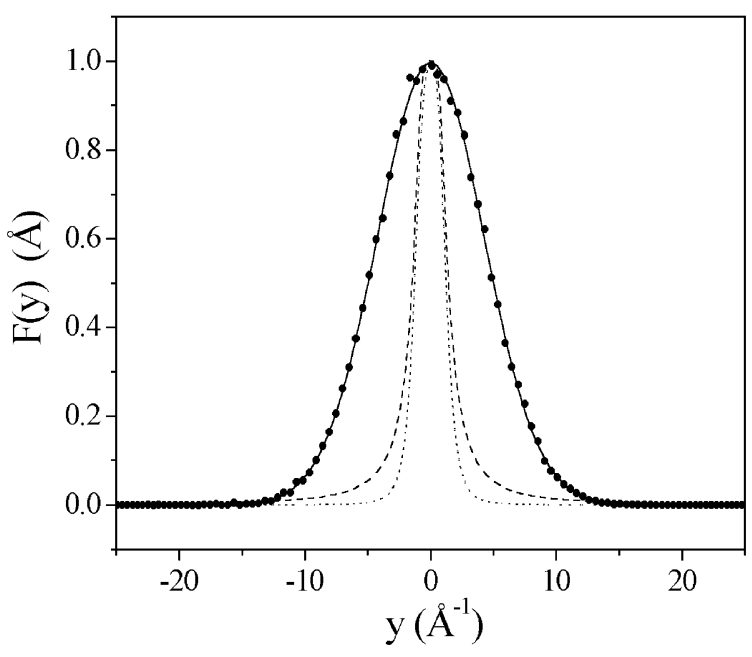

Fig. 6. Simulated DD response function, $F(y)$, for $\mathrm{H}$ in $\mathrm{ZrH}_{2}$ (full circles), obtained using ${ }^{238} \mathrm{U}$ filters at room temperature and the corresponding fit according to Eq. (26) (full line). Simulation refers to a scattering angle $2 \vartheta_{n}=42.54^{\circ}$. Instrument resolution for the SD and DD methods, is represented by dashed and dotted lines, respectively.

The analysis developed below aims to predict the effectiveness of the DD method in deriving information on the single particle momentum distribution from the experimental response function. After producing the simulated data for $\mathrm{Pb}$ and $\mathrm{ZrH}_{2}$, we have fitted them in order to extract the standard deviation of the proton momentum distribution in $\mathrm{ZrH}_{2}$, i.e., $\sigma_{\mathrm{H}}$, which has to be compared with the initial value inserted in the DINSMS Monte-Carlo code used for this simulation itself. The rationale behind our data analysis is to reduce as much as possible the approximations concerning the resolution function. In this view, we made use of the following fitting functions in $y$ space:

$$
\begin{aligned}
F_{\mathrm{Pb}}^{\mathrm{SD}}(y)= & A\left\{G_{\mathrm{geom}}\left(y, \sigma_{\mathrm{g}}\right)\right. \\
& \left.\otimes X_{\mathrm{SD}}(y) \otimes J_{\mathrm{Pb}}\left(y, \sigma_{\mathrm{Pb}}\right)\right\} \\
F_{\mathrm{Pb}}^{\mathrm{DD}}(y)= & A\left\{G_{\mathrm{geom}}\left(y, \sigma_{\mathrm{g}}\right)\right. \\
& \left.\otimes X_{\mathrm{DD}}^{\beta}(y) \otimes J_{\mathrm{Pb}}\left(y, \sigma_{\mathrm{Pb}}\right)\right\} \\
F_{\mathrm{H}}^{\mathrm{DD}}(y)= & A\left\{G_{\mathrm{geom}}\left(y, \sigma_{\mathrm{g}}\right)\right. \\
& \left.\otimes X_{\mathrm{DD}}^{\beta}(y) \otimes J_{\mathrm{H}}\left(y, \sigma_{\mathrm{H}}\right)\right\}
\end{aligned}
$$


where

$$
\begin{aligned}
X_{\mathrm{SD}}(y)= & 1-\exp \left[-b \bar{f}\left(y, \Gamma_{0}, \sigma_{T}\right)\right] \\
X_{\mathrm{DD}}^{\beta}(y)= & X_{\mathrm{SD}}(y) \\
& \quad-\beta\left\{1-\exp \left[-\frac{b}{\beta} \bar{f}\left(y, \Gamma_{0}, \sigma_{T}\right)\right]\right\}
\end{aligned}
$$

are the transfer functions of the spectrometer in the $\mathrm{SD}$ and in the $\mathrm{DD}$ case, $G_{\text {geom }}\left(y, \sigma_{\mathrm{g}}\right)$ is the geometrical component of the resolution function in the $y$ space and $J_{\mathrm{H}(\mathrm{Pb})}\left(y, \sigma_{\mathrm{H}(\mathrm{Pb})}\right)$ is the Gaussian compton profiles for a hydrogen (lead) nucleus. Symbols $A$ and $b$ denote fitting parameters. Eqs. (24)-(26) represent a substantial refinement with respect to the usual fitting procedure [19], which indeed considers the transfer function of the instrument, for a sufficiently thin foil, as the result of the following approximation:

$$
\begin{aligned}
X_{\mathrm{SD}}(E) & =1-T(E) \\
& =1-\exp [-\tau \bar{f}(E)] \simeq \tau \bar{f}(E) .
\end{aligned}
$$

Moreover the energy resolution function of an infinitely thin ${ }^{238} \mathrm{U}$ foil, which has actually a Voigt line shape (see Eq. (10)), is often replaced by a simple Gaussian Doppler component, neglecting the minor contribution from the intrinsic Lorentzian energy component.

Eq. (24) has been used to fit the SD lead spectrum in order to determine the $\Gamma_{0}$ and $\sigma_{\mathrm{g}}$ parameters, while the standard deviation of the Doppler contribution, $\sigma_{T}$, and of the lead momentum distribution, $\sigma_{\mathrm{Pb}}$, have been fixed to their known values. The obtained resolution components are dependent, in $y$ space, on the mass of the nuclei and on the momentum transfer, $\hbar q$, at the top of the inelastic peak $(y=0)$. For instance the quantity $\sigma_{\mathrm{g}}$, calibrated using the $\mathrm{Pb}$ sample, can be approximately expressed in the proton $y$ space through

$\sigma_{\mathrm{g}, M_{\mathrm{H}}} \simeq \frac{M_{\mathrm{H}} q_{\mathrm{Pb}}}{M_{\mathrm{Pb}} q_{\mathrm{H}}} \sigma_{\mathrm{g}, M_{\mathrm{Pb}}}$

where $M_{\mathrm{H}}, M_{\mathrm{Pb}}$ are the recoiling masses, and $q_{\mathrm{H}}, q_{\mathrm{Pb}}$ the corresponding values of the momentum transfer at $y=0$ [33]. Once these resolutions components are transformed and inserted into Eq. (26), which is the complete expression for the response function of the proton when DD is used, the only varying parameters in the fit are the standard deviation $\sigma_{\mathrm{H}}$ and the two factors $A$ and $b$. The fitting procedure, accomplished by an FORTRAN code, making use of the MINUIT minimisation routine [34] yielded a single $\sigma_{\mathrm{H}}$ value of $(4.10 \pm 0.05) \AA^{-1}$, to be compared with the value inserted in the Monte-Carlo simulation, namely $4.14 \AA^{-1}$. The quality of the fit is shown in Fig. 6 , together with the SD and DD resolution functions and confirms the validity of the convolution approximation, since the Monte-Carlo code does not involve analytic convolutions of the transfer functions of the single components of the apparatus (sample, filter, detectors, etc.). In Fig. 6 the decrease of the HWHM in the DD case is clear. Furthermore, the DD method gives rise to a remarkable change of the line shape of the instrumental resolution function $R_{n}(y)$. To make quantitative this statement, let us define, both for the DD case and for SD case with thin uranium foil $(\tau=1.8)$, the quantities

$$
\begin{aligned}
& P=\int_{0}^{\left|y_{1}\right|} R_{n}(y) \mathrm{d} y \\
& T=\int_{\left|y_{1}\right|}^{\infty} R_{n}(y) \mathrm{d} y
\end{aligned}
$$

where $n$ labels the detectors at the scattering angle $2 \vartheta_{n}$ and $y_{1}$ is the value of the scaling variable corresponding to the HWHM. The ratio $\zeta=P / T$ is an estimate of the weight of the area around the peak with respect to the area of the tails. The values, $\zeta=1.49$ and 3.00 are retained for the SD and DD methods, respectively. This means that the DD method lowers the weight of the tails, mainly produced as an effect of the intrinsic Lorentzian component of the resolution, by a factor of two with respect to SD method.

\subsection{Simulated DINS experiments with $\gamma$ detectors}

\subsubsection{Resonance Detector technique principles}

Very recently the Resonance Detector (RD) technique [21] has been reconsidered for DINS experiments [20,24], since it makes possible to extend the accessible region of the kinematic space $(\hbar q, \hbar \omega)$. As a matter of fact, the efficiency of the ${ }^{6} \mathrm{Li}$-glass scintillation detectors presently used on 
eVS and VESUVIO, is low at high neutron energies, $E_{\mathrm{n}}$, because of the $1 / \sqrt{E_{\mathrm{n}}}$ functional dependence of the neutron capture cross-section. In $\mathrm{RD}$ technique the detection of the scattered neutrons, is obtained by revealing the prompt $\gamma$-ray cascade, which follows the resonant absorption of neutrons by the filters. The detection efficiency is independent of the neutron energy at a high degree of accuracy. Furthermore, employing filters with a good yield for low energy $\gamma$, such as ${ }^{197} \mathrm{Au}$ and ${ }^{238} \mathrm{U}$, and proper detectors, it is possible to reach appreciable improvements of the signal to background ratio. A detailed description of this technique is presented in Refs. [20,24]. A comparison between simulated and experimental results obtained on eVS/VESUVIO spectrometer for a lead reference sample in the $\mathrm{RD}$ technique configuration, is presented in this section. Specifically, the experimental setup involved the $E_{0}=6.671 \mathrm{eV}$ resonance of the ${ }^{238} \mathrm{U}$ filter.

\subsubsection{Simulation}

An RD-DINS experiment has been simulated using a properly modified version of the DINSMS code. As remarked above, in the RD approach the scattered neutrons are selectively detected by revealing the $\gamma$-ray cascade, promptly produced after neutron radiative capture by the filters foils. In the radiative neutron capture process a neutron with energy $E_{\mathrm{n}}$ interacts with a target nucleus of mass $A$ and a compound nucleus of mass $(A+1)$ is formed, whose recoil energy $E_{(A+1)}$ is generally negligible. A discrete emission of photons is also produced, the so-called $\gamma$ cascade. The crosssection of this nuclear process, shown in Fig. 7 for the ${ }^{238} \mathrm{U}$, can be described in terms of the single level Breit-Wigner resonance formalism (see Eq. (7)). The energy released by the reaction is mostly emitted in form of electromagnetic radiation, so that the neutron capture event can be effectively detected by revealing the prompt $\gamma$-ray cascade. A good absorber has to fulfill the following conditions:

(1) the radiative capture cross-section should have intense and well-isolated narrow peaks in the energy range of interest $(1-100 \mathrm{eV})$;

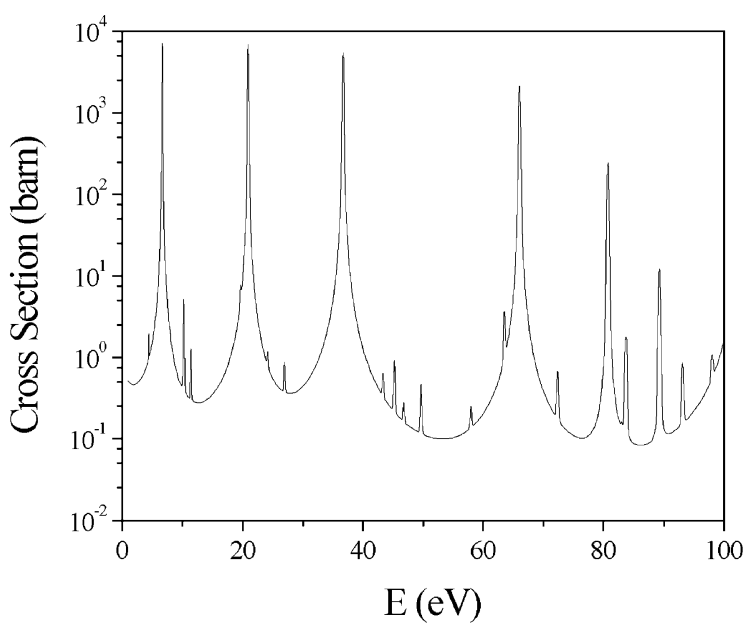

Fig. 7. Radiative capture cross-section of ${ }^{238} U$ in the energy interval $0.01-100 \mathrm{eV}$ [36].

(2) the yield for the low energy $\gamma$-ray emission has to be high.

The first requirement is important both to improve the spectrometer resolution and to insure the validity of the approximations used in Section 3. The second requirement is related to the possibility of enhancing the signal-to-background ratio by using $\gamma$ detectors with a small effective volume and good efficiency for low energy $\gamma$ radiation. In the epithermal energy range of interest, several materials fit the above conditions [24], e.g., ${ }^{238} \mathrm{U}$ and ${ }^{197} \mathrm{Au}[35,36]$.

In the Monte-Carlo code the neutron detection process through ${ }^{6} \mathrm{Li}$-glass has been substituted by $\gamma$-ray production in the absorber and $\gamma$-ray detection through $\mathrm{NaI}(\mathrm{Tl})$ scintillator.

In this simulation the filter thickness values $\tau_{1}=$ 1.1 , corresponding to a thin foil $30 \mu \mathrm{m}$ thick of ${ }^{238} \mathrm{U}$, and $\tau_{2}=4.4$, corresponding to a thick foil $120 \mu \mathrm{m}$ thick, both at $T=300 \mathrm{~K}$, have been employed. These values give rise to the foil thickness ratio $\beta=0.28$ and are the ones effectively used during the experimental measurements.

As in the previous simulation (see Section 4.1), outputs are time-of-flight spectra. As far as the signal intensity is concerned, we assumed a unitary and energy independent efficiency for the $\gamma$ cascade production, so that the number of 
produced cascades equals the number of scattered neutrons absorbed in the ${ }^{238} \mathrm{U}$ foil. This assumption is reasonable, considering that the radiative channel branching ratio is the highest around $E_{0}$ and that it varies less than $5 \%$ within the width of the resonance, $\Gamma_{0}=55 \mathrm{meV}$ at room temperature, as shown in Fig. 8.

The correct count of the absorbed neutron number depends on the number of $\gamma$ cascades effectively revealed by the scintillator and then on the efficiency $\eta(\varepsilon)$ of the $\gamma$ detector ( $\varepsilon$ being the $\gamma$ energy). In this context, as no $\gamma$ spectroscopic investigation was needed, the scintillator has been considered as a calorimeter, so that the mean total energy deposit of each cascade within the detector volume is the important quantity for the complete determination of the number of absorbed neutrons. In our simulation a constant effective efficiency, $\eta=1$, has been considered. In fact, any proportional change in the number of scintillation photons produced in the $\mathrm{NaI}(\mathrm{Tl})$, would result in a scale factor in the final signal, without changing the response shape. This can be originated by a partial detection of the $\gamma$ cascade energy deposit, resulting from an energy-dependent detector efficiency $\eta\left(\varepsilon_{i}\right)$ or a finite size detector geometrical acceptance. The neutron time of flight from the moderator to the ${ }^{238} \mathrm{U}$ foil has been determined by using the $\gamma$ detection as stop signal,

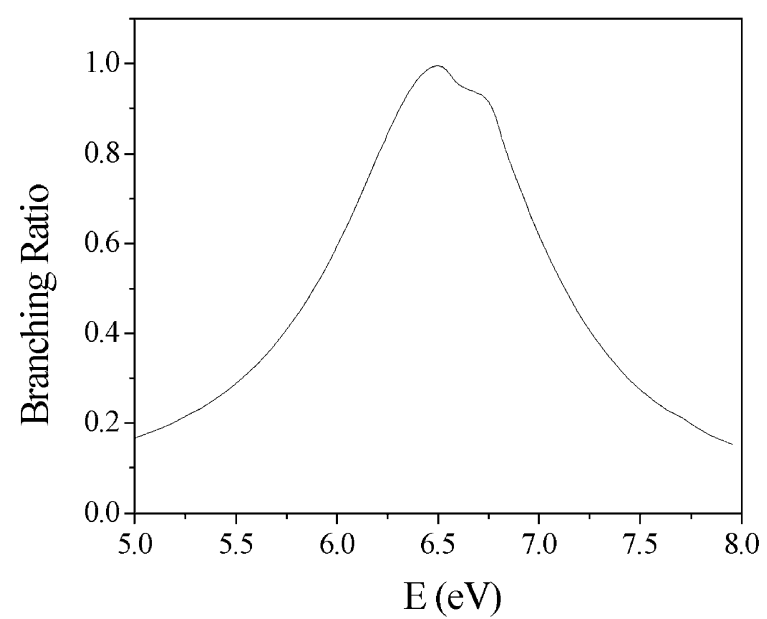

Fig. 8. Branching Ratio (BR) of the radiative channel in an energy interval centred around the ${ }^{238} \mathrm{U}$ resonance at $6.67 \mathrm{eV}$. assumed coincident with neutron absorption in the $\mathrm{U}$ foil (reasonable assumption, since the $\gamma$ cascade is prompt). An overall time offset $t_{\text {off }} \simeq 1 \mu \mathrm{s}$, mostly due to the electromagnetic signal acquisition chain (NaI-Photomultiplier tube-transmission

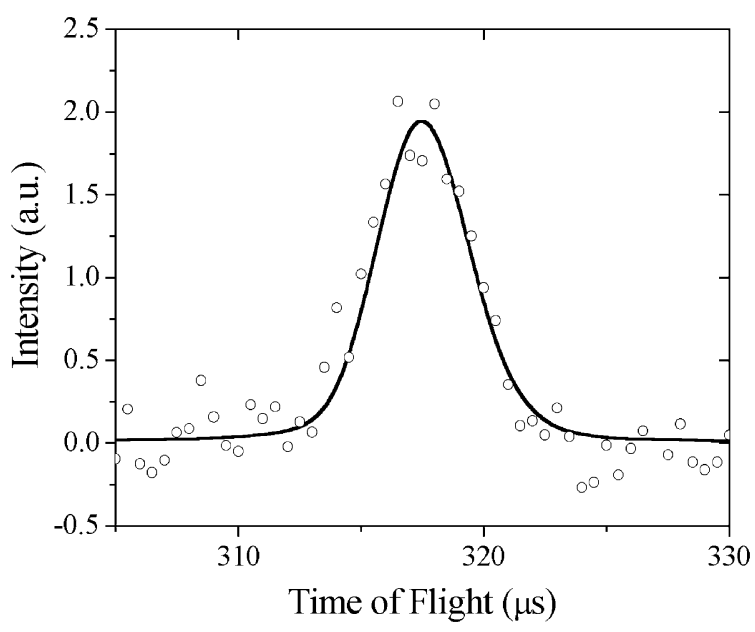

Fig. 9. Simulated (full line) and experimental (open circles) SD recoil spectra of $\mathrm{Pb}$ relative to the $6.67 \mathrm{eV}$ resonance of a ${ }^{238} \mathrm{U}$ foil at room temperature. The experimental recoil spectrum has been acquired using $\mathrm{NaI}(\mathrm{Tl})$ scintillators at the scattering angle $29=38^{\circ}[20]$.

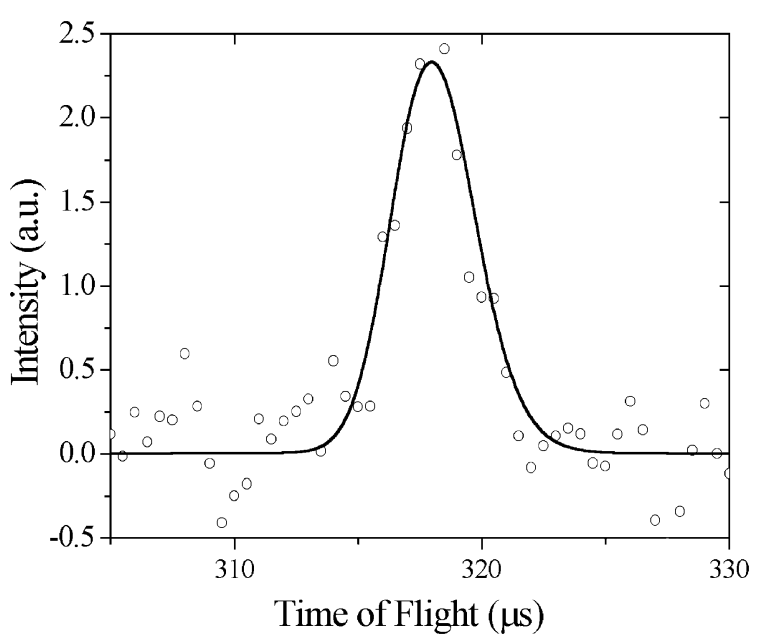

Fig. 10. Simulated (full line) and experimental (open circles) $\mathrm{DD}$ recoil spectra of $\mathrm{Pb}$ relative to the $6.67 \mathrm{eV}$ resonance of a ${ }^{238} \mathrm{U}$ foil at room temperature. The experimental recoil spectrum has been acquired using $\mathrm{NaI}(\mathrm{Tl})$ scintillators at the scattering angle $2 \vartheta_{n}=38^{\circ}$ [20]. 
cables, electronic modules-computer) has been introduced.

DINS exploratory data employing RD technique with NaI detectors are available in Ref. [20]. These data were obtained at eVS spectrometer on a polycrystalline lead sample at room temperature for $29 \simeq 38^{\circ}$. In Ref. [20], it has been stressed that the DD method allows a sensible improvement of the spectrometer resolution with respect to the SD method. In Figs. 9 and 10 the SD and DD recoil spectra for lead, both simulated and experimental, obtained with a ${ }^{238} \mathrm{U}$ foil at room temperature are shown in the time-of-flight region corresponding to $6.67 \mathrm{eV}$ scattered neutrons. In both cases, the simulated data have been corrected for the estimated time offset. As it can be argued from these figures, the agreement between experimental data and simulations appears satisfactory, in spite of the low data statistics.

\section{Discussion and conclusions}

The dependence on the parameters $\beta$ and $\tau$ of the HWHM, $\Gamma_{\mathrm{DD}}(\beta, \tau)$, for DD transfer function is shown in Figs. 1-3 for various filters and different temperatures. In Fig. $1 \Gamma_{\mathrm{DD}}(\beta, \tau)$ is calculated in the zero- $T^{*}$ limit, i.e., without temperature effects, and it is referred to as $\Gamma_{\mathrm{DD}}^{0}(\beta, \tau)$ (see Section 3.2). Calculations are shown as a function of the dimensionless thickness, $\tau$, for both $\beta=0$ and 1 . It is worthwhile to remind that the $\beta=0$ case coincides with the single difference HWHM, while $\beta=1$ corresponds to the ideal case of two filters of identical thickness. The real cases of intermediate $\beta$ values occur in between the two lines plotted in Fig. 1. The presence of a limiting value for the resolution improvement using the DD method is clearly evident when $\tau \simeq 0$ and $\beta \simeq 1$. As far as Fig. 2 is concerned, the same quantities of Fig. 1 are reported, but the Doppler broadening being taken into account. Since the relative influence of the Doppler broadening is filter dependent, two different foils have been selected: liquid nitrogen temperature ${ }^{238} \mathrm{U}$ (squares) and room temperature ${ }^{197} \mathrm{Au}$ (circles), together with the two extreme values of $\beta$, namely $\beta=1$ (empty symbols) and $\beta=0$ (full symbols). The ratio $\alpha=\Gamma_{\mathrm{DD}}(\beta=1$, $\tau=0) / \Gamma_{\mathrm{DD}}(\beta=0, \tau=0)$ is equal to $\Gamma_{\mathrm{DD}}(\beta=$ $1, \tau=0) / \Gamma_{\mathrm{SD}}(\tau=0)$ (see Fig. 2) has values of 0.69 and 0.67 for ${ }^{238} \mathrm{U}$ at liquid nitrogen temperature, and for ${ }^{197} \mathrm{Au}$ at room temperature, respectively. It should be noted that these values of the ratio $\alpha$ at room temperature are both only slightly higher than the value at zero effective-temperature (i.e., 0.64). The ratio between the DD HWHM, $\Gamma_{\mathrm{DD}}(\beta, \tau)$, and the resonance Lorentzian HWHM, $\Gamma_{0}$, as a function of the parameter $\beta$ is shown in Fig. 3. The calculations are reported for two different ${ }^{238} U$ filter configurations: at room (squares) and liquid nitrogen temperature (circles), corresponding to $\tau=1.1$ and 1.6, respectively. Room temperature ${ }^{197} \mathrm{Au}$ data (triangles) corresponding to $\tau=1.8$ are also plotted. It is clear that there is only little improvement in the resolution for $\beta \geqslant 0.3$, independently of the nature and the temperature of the filter.

Another key element for assessing the applicability of the DD method is the counting statistics, i.e., the acquisition time. In Section 3.3 we have introduced the useful dimensionless parameter $R$, that is the time factor required by each separate measurement in order to obtain the same statistical accuracy achieved in an SD spectrum. In Fig. 4(a) we have shown the ratio $R$ as a function of $\beta$ for two values of the dimensionless thickness $\tau$, namely $\tau=1$ (full line) and $\tau=2$ (dotted line). It is evident that for large $\beta, R$ increases quite rapidly values towards the expected divergence at $\beta=1$. As it has been stressed before, $\beta=1$ is the unphysical case where the two foils have the same thickness and no statistics in the final DD spectrum can be built up. In Fig. 4(b) the same concept is made even clearer. The linear relationship between the duration of an experiment on VESUVIO spectrometer employing the DD method and the one from experiment employing the SD method, within the same statistical accuracy, is plotted for three different couples of $\beta$ and $\tau$ values: $\beta=0.4, \tau=1$ (circles); $\beta=0.4, \tau=1.5$ (triangles) and $\beta=0.28, \tau=1.8$ (squares). From Figs. 1, 2 and 4 one can notice that also for the thickness parameter $\tau$, as well as for the parameter $\beta$, a compromise has to be found. Indeed, a smaller value of $\tau$ yields a better resolution, but increases the collection time. 
The results of DINSMS Monte-Carlo simulated experiments for ${ }^{6} \mathrm{Li}$-glass scintillators with natural uranium filter foils at room temperature are shown in Figs. 5 and 6. In Fig. 5, the normalised SD (full circles) and DD (open circles) data for the calibration polycrystalline $\mathrm{Pb}$ sample at room temperature are reported. The DD method gives a remarkable resolution improvement and a sensible increased contrast (i.e., decreased intensity in the tails). This occurs even for the uranium filter, as in the present case, where the slowly decaying Lorentzian contribution, $\left(E-E_{0}\right)^{-2}$, to the line shape is dominated by the Doppler Gaussian one.

In Fig. 6 the DD response function for a $\mathrm{ZrH}_{2}$ sample at room temperature is reported. The best fit of simulated data obtained according to Eq. (26) (full dots) is represented by the full line. The instrument resolution for SD and DD methods, represented by dashed and dotted lines, respectively, are also shown. The quality of the fit confirms the validity of our approach, where: (i) the refined expressions of Eqs. (24)-(26) are used, instead of the approximation $X_{\mathrm{SD}}(E) \simeq \tau \bar{f}(E)$, as explained in Section 2; (ii) the full Voigt line shape is used for the energy resolution function (see Eq. (10)), instead of the simple Gaussian Doppler component, which neglects the contribution of the intrinsic Lorentzian energy component. It is worth noting that these results are also a confirm of the applicability of the convolution approximation. From a comparison between SD and DD lead response functions, obtained in the RDS approach (see Figs. 9 and 10), the resolution improvement attainable with the DD method can be appreciated also within the RDS detection technique.

In conclusion, we have shown that the improvement of the spectrometer resolution due to the application of DD method is quite remarkable. The improvement of the DD method is twofold. On one hand, the HWHM is reduced, as illustrated in Section 3; on the other hand, the weight of the tails in the line shape of the instrument resolution function is lowered by a factor of two, as compared to the SD method (see Section 4.1), and this effect can allow a more accurate measurement of $\left\langle E_{\mathrm{k}}\right\rangle$.

We have also proved for the first time that the DD method has an intrinsic limitation in reducing the HWHM of the effective transfer function of an inverse geometry spectrometer, as expressed by Eq. (18). Indeed, the line width can be reduced at most by a factor $\sqrt{\sqrt{2}-1}$ in the zero effectivetemperature limit and in the unachievable case of $\beta=1$. Nevertheless, we find that if the Doppler effect due to thermal motion is taken into account and values of $\beta$ yielding an affordable expansion in the required counting times are used, this factor is only slightly increased so that a realistic reduction factor of about 0.75 can be achieved, using the most popular filters (see Figs. 3 and 4). Efforts to get closer to the ultimate limit are, in our opinion, not rewarding because of an excessive counting time. However, it must be kept in mind that another advantage of the DD method is to largely remove the Lorentzian tails in the transfer function (increased contrast), which in the limiting case of Eq. (17) can be described (similarly to a double grating monochromator) by squaring the SD transfer function.

The simulation results presented in this paper are obtained from a completion of the DINSMS code and confirm that this code represents an extremely useful tool for defining the more suitable instrumental arrangement for each experiment on VESUVIO spectrometer in the standard RFS setup both within the SD and DD approaches.

It has to be mentioned that the experimental activity for the development and improvement of neutron detection techniques in neutron spectroscopy at the eV energy region is presently matter of great interest. In particular, the advantages of the RDS set-up, as compared to standard RFS one, are appreciable as far as detection efficiency at high neutron energies and signal to background ratio are concerned [20]. In this context, the present results demonstrate that the DINSMS code is able to properly describe the experimental data when both RDS approach and DD method are employed.

\section{References}

[1] M. Celli, M. Zoppi, J. Mayers, Phys. Rev. B 58 (1998) 242; R. Senesi, C. Andreani, D. Colognesi, A. Cunsolo, M. Nardone, Phys. Rev. Lett. 86 (2001) 4584. 
[2] D.A. Peek, I. Fujita, M.C. Schmidt, R.O. Simmons, Phys. Rev. B 45 (1992) 9680;

M.A. Fradkin, S.X. Zeng, R.O. Simmons, Phys. Rev. 49 (1994) 3197;

D.A. Peek, M.C. Schmidt, I. Fujita, R.O. Simmons, Phys. Rev. B 45 (1992) 9671.

[3] C. Andreani, E. Degiorgi, R. Senesi, F. Cilloco, D. Colognesi, J. Mayers, M. Nardone, E. Pace, J. Chem. Phys. 114 (2001) 387;

C. Andreani, D. Colognesi, E. Degiorgi, M.A. Ricci, J. Chem. Phys. 115 (2001) 11243.

[4] S. Fulton, R.A. Cowley, A.C. Evans, J. Phys: Condens. Matter 6 (1994) 2977;

A.C. Evans, J. Mayers, D.N. Timms, J. Phys: Condens. Matter 6 (1994) 4197

[5] W.E. Langel, D.L. Price, R.O. Simmons, P.E. Sokol, Phys. Rev. B 38 (1988) 275;

C. Andreani, D. Colognesi, E. Pace, Phys. Rev. B 60 (1999) 10008.

[6] F.J. Bermejo, F.J. Mompean, A. Srinivasan, J. Mayers, A.C. Evans, Phys. Lett. A 189 (1994) 333;

J. Dawidowski, F.J. Bermejo, L. Fernandez Barquin, P. Gorria, J.M. Barandarian, A.C. Evans, J. Mayers, Phys. Lett. A 214 (1996) 59.

[7] M.P. Pauli, R.S. Holt, J. Phys. C: Solid State Phys. 21 (1988) 3633;

A.L. Fielding, D.N. Timms, J. Mayers, Europhys. Lett. 44 (1998) 255.

[8] E.B. Karlsson, C.A. Chatzidimitriou-Dreismann, T. Abdul-Redah, R.M.F. Streffer, B. Hjörvarsson, J. Öhrmalm, J. Mayers, Europhys. Lett. 46 (1999) 617.

[9] A.C. Evans, J. Mayers, D.N. Timms, M.J. Cooper, Z. Naturforsch. Teil A 48 (1993) 425.

[10] C.G. Windsor, Pulsed Neutron Scattering, Taylor and Francis, London, 1981.

[11] B.E.F. Fender, et al., Phyl. Trans. R. Soc. B 290 (1980) 657.

[12] P.C. Hohenberg, P.M. Platzman, Phys. Rev. 152 (1966) 198.

[13] S.W. Lovesey, Theory of Neutron Scattering from Condensed Matter, Oxford University Press, London, 1987.

[14] B. Williams, Compton Scattering, McGraw-Hill, New York, 1977.

[15] C. Ciofi degli Atti, E. Pace, G. Salmè, Phys. Rev. C 43 (1991) 1155.

[16] A.L. Fielding, D.N. Timms, J. Mayers, Europhys. Lett. 44 (1998) 255.
[17] R. Senesi, C. Andreani, Z. Bowden, D. Colognesi, E. Degiorgi. A.L. Fielding, J. Mayers, M. Nardone, J. Norris, M. Praitano, N.J. Rhodes, W.G. Stirling, J. Tomkinson, C. Uden, Physica B 276-278 (2000) 200.

[18] P.A. Seeger, A.D. Taylor, R.M. Brugger, Nucl. Instr. and Meth. A 240 (1985) 98.

[19] J. Mayers, A.C. Evans, Rutherford Appleton Laboratory Report RAL-91-048, 1991.

[20] C. Andreani, A. Pietropaolo, R. Senesi, G. Gorini, M. Tardocchi, A. Bracco, N. Rhodes, E.M. Schooneveld, Nucl. Instr. and Meth. A 481 (2002) 509.

[21] J.M. Carpenter, N. Watanabe, S. Ikeda, Y. Masuda, S. Sato, Physica B 120 (1983) 126.

[22] N. Watanabe, IAEA-CN-46/26, 1985, p. 279.

[23] D.R. Allen, E.W.J. Mitchell, R.N. Sinclair, J. Phys. E: Sci. Instrum. 13 (1980) 639.

[24] A. Pietropaolo, C. Andreani, A. D’Angelo, R. Senesi, G. Gorini, S. Imberti, M. Tardocchi, N. Rhodes, E.M. Schooneveld, Appl. Phys. A 74 (2002) 1.

[25] G.B. West, Phys. Rep. 18 (1975) 263; G. Watson, J. Phys: Condens. Matter 8 (1996) 5955; E. Pace, G. Salme', G. West, Phys. Lett. B 273 (1991) 205.

[26] C. Andreani, G. Baciocco, R.S. Holt, J. Mayers, Nucl. Instr. Meth. A 276 (1991) 205.

[27] J.J. Blostein, J. Dawidowski, J.R. Granada, Physica B 304 (2001) 357.

[28] C. Andreani, D. Colognesi, A. Filabozzi, E. Pace, M. Zoppi, J. Phys: Condens. Matter 10 (1998) 7091.

[29] C. Andreani, E. Degiorgi, M. Nardone, R. Senesi, Internal Report Rom2F/98/04, 1998.

[30] V.F. Turkin, Slow Neutrons, Jerusalem, 1965 (Israel Program for Scientific Translations).

[31] J. Mayers, A.L. Fielding, R. Senesi, Nucl. Instr. and Meth. A 481 (2002) 258.

[32] A.C. Evans, D.N. Timms, J. Mayers, S.M. Bennington, Phys. Rev. B 53 (1996) 3023;

C. Andreani, A. Filabozzi, E. Pace, J. Mayers, Phys. Rev. B 54 (1996) 6255.

[33] D. Colognesi, Ph.D. Thesis, Università degli Studi di Roma "Tor Vergata", 1997, unpublished.

[34] F. James, MINUIT minimization package: reference manual, CERN Program Library, Geneva, 1994.

[35] J. Mayers, C. Andreani, D. Colognesi, J. Phys: Condens. Matter 9 (1997) 10639.

[36] S.F. Mughabghab, M. Divadeenam, N.E. Holden, Neutron Cross Sections, Vol. I, Part A, Academic Press, New York, 1981. 